\title{
Correr, sufrir, disfrutar. El cuerpo como instrumento de investigación
}

\author{
Running, suffering, enjoying. The body as a research instrument \\ Gastón Julián Gil \\ CONICET - Universidad Nacional de Mar del Plata, Argentina \\ gasgil@mdp.edu.ar
}

\section{RESUMEN:}

El running constituye un estilo de vida de notoria masividad en la Argentina contemporánea que excede la decisión puntual de salir a correr con frecuencia de manera solitaria o en grupos de entrenamiento. En este caso, la decisión de realizar una etnografía del fenómeno obliga al investigador a compartir las rutinas y exigencias habituales de actores que se imponen desafíos variados para completar, en el mejor tiempo posible, carreras de larga distancia. Así es que un etnógrafo, como un nativo marginal, necesita mantener un estado atlético que le permita sostener algunas de esas exigencias que además constituyen instancias fundamentales del trabajo de campo. Por ende, en este artículo se analiza al cuerpo del etnógrafo como un instrumento clave de investigación, apelando a diversas herramientas teóricas, principalmente fenomenológicas. Aunque no se plantea que este grado de involucramiento corporal sea excluyente, sí implica un recurso relevante para comprender un estilo de vida caracterizado por el desarrollo de firmes preceptos morales y estéticos. Esta fenomenología del etnógrafo-corredor permite entonces describir, a través de la conciencia encarnada, un amplio conjunto de emociones y "técnicas corporales" que son definitorias de la pasión por correr como un estilo de vida. Todo ello está orientado a desarrollar principios metodológicos que hacen a la producción del conocimiento social y que trascienden a este tipo de prácticas deportivas.

Palabras ClaVe: Emociones, Running, Etnografía, Embodiment.

\begin{abstract}
:
Running constitutes a lifestyle of notorious massiveness in contemporary Argentina. It goes beyond the specific decision to go running frequently, alone or in training groups. In this case, the decision to carry out an ethnographic research of the running phenomenon forces the scientist to share the usual routines and demands of the actors who face different challenges to complete long-distance races in the best possible time. Thus, the ethnographer, as a marginal native, needs to maintain a reasonably good physical state in order to be able to satisfy some of the demands that also constitute fundamental instances of the fieldwork. Therefore, in this article, the body of the ethnographer is analyzed as a key research in terms of various theoretical resources, mainly phenomenological techniques. Although the article does not state that this degree of bodily involvement is a necessary condition, it does propose that it is a fundamental resource for understanding a particular lifestyle that is characterized by the development of firm moral and aesthetic precepts. This phenomenology of the ethnographer-runner then enables us to describe, by means of embodied consciousness, a broad set of emotions and "body techniques" that define the passion for running as a lifestyle. It is aimed at developing methodological principles that lead to the production of social knowledge and that transcend sports practices like running.
\end{abstract}

KEYWORDS: Emotions, Running, Ethnography, Embodiment.

\section{INTRODUCCIÓN}

La masiva adhesión al running en tanto pasión globalizada de sujetos que no sólo salen a correr, configura un fenómeno de gran relevancia en las sociedades contemporáneas. En países como la Argentina, y más precisamente en ciudades como Mar del Plata, donde se lleva a cabo la mayor parte del trabajo de campo, la explosión del running puede constatarse en costaneras y boulevards, plazas y demás paseos urbanos que se pueblan todos los días, y durante cualquier horario, de corredores solitarios y grupales. En su gran mayoría no son atletas federados, algunos tal vez lo hacen como complemento aeróbico para la práctica de otros deportes,

\section{Recepción: 10 Mayo 2020 | Aprobación: 20 Septiembre 2020 | Publicación: 30 Septiembre 2020}


pero la mayor parte de ellos son runners y cumplen con sus entrenamientos junto con otros miembros de sus running teams. El diseño de una etnografía sobre el colectivo runner coloca al investigador ante el desafío de no sólo involucrar su cuerpo de manera particular en el proceso sino que además se impone una reflexión minuciosa sobre ese compromiso corporal que demanda una exigencia física considerable. De todos modos, este enfoque autoetnográfico de la fenomenología del investigador que corre constituye una herramienta metodológica destinada a una mejor comprensión del running y no una coartada para una antropología autorreferencial. Por supuesto, no se formula en este artículo que esta opción metodológica sea indispensable para producir conocimiento sobre los corredores pero sí que ofrece recursos relevantes para comprender este estilo de vida.

La adopción de este estilo de vida suele producir en sus aficionados diversos cambios en su modo de vida pero también en su configuración corporal. Ello se enmarca en un contexto global en el que "para muchos aficionados, un elemento importante del running consiste en la ayuda que proporciona para mejorar su propio capital físico y su atractivo, lo que implica una inversión sobre el propio cuerpo (Shipway \& Holloway, 2016, p. 274). Esa inversión no necesariamente se traduce en el logro de un "cuerpo perfecto", más allá de que la práctica deportiva favorezca claramente la conformación de un cuerpo esbelto, "contenido, restringido y controlado emocionalmente" (Atkinson, 2008: 176), sobre todo en deportes de exigencias extraordinarias. De un modo un tanto un tanto extremo, Abbas (2004) plantea que el cuerpo delgado y tonificado constituye un ideal para personas de todas las edades y en particular para las "clases medias", tanto por motivos de salud como estéticos, y cuyos efectos principales consisten en naturalizar las desigualdades de género, etarias y de clase, al individualizar la responsabilidad por el cuidado del cuerpo y la salud. En contraposición a estas lecturas lineales, la experiencia de campo más elemental muestra una frecuencia alta de cuerpos más voluminosos de muchos corredores que desafían los imaginarios dominantes, ${ }^{1}$ más allá de las eventuales estigmatizaciones que indicarían falta de autodisciplina, por ejemplo en las comidas (Chase, 2008). Precisamente, un indicador de los modos más "serios" (Shipway \& Holloway, 2013) de apropiación del running es la adopción de pautas de alimentación estrictas, que suelen ser resumidas en una alimentación que busca obtener un mejor rendimiento a partir de un cuerpo más esbelto y tonificado y, por consiguiente, un "mejor" estado de salud. Esta alimentación "saludable" y "natural", propia de la "cultura fit" (Hijós, 2017) rechaza la ingestión de grasa animal, embutidos, harinas blancas, hidratos de carbono y comidas con conservantes. Esta opción puede implicar el abandono del consumo de carne o de cualquier alimento de origen animal (como los huevos o los lácteos). Como han mostrado Shipway \& Holloway (2016), los corredores de larga distancia ${ }^{2}$ "no solo adaptan el peso corporal y la figura a las normas culturales de la comunidad en general, sino también a las de su propia subcultura" (Shipway \& Holloway, 2016, p. 90) porque además "necesitan hacer del cuerpo el instrumento a través del cual logran éxito" (Shipway \& Holloway, 2016, p. 90). Estos "éxitos" son definidos subjetiva e intersubjetivamente para cada itinerario y cada contexto, por lo que pueden referir a resultados deportivos (un tiempo, una posición), corporales (perder peso) o psicológicos (sentirse mejor), entre otras posibilidades que permiten los amplios márgenes de apropiación diferencial que caracterizan a este estilo de vida (Gil, 2018).

Así es que en este artículo se desarrolla la relevancia de una reflexión sobre la fenomenología del etnógrafo, en este caso en tanto nativo marginal. Esta autodefinición remite a un investigador que pese a compartir marcos, experiencias y ciertas representaciones con sus sujetos de estudio, nunca se percibió como un runner, sino como alguien que sólo sale a correr, que se inscribe en algunas carreras, que no mantiene rutinas de entrenamientos constantes y que además no sigue las dietas estrictas y planificadas que caracterizan a los corredores "serios". Esta autocategorización como nativo marginal se fue conformando mucho antes de definir al running como tema de investigación. La distancia crítica con el modo en que los aficionados estaban transformando la práctica de salir a correr nunca dejó de provocar resonancias y hasta cierto rechazo. Ello permitió multiplicar exponencialmente el extrañamiento mientras que junto con el placer por correr se iban incorporando rutinas y sobre todo, algunos de los principales rituales de interacción, las carreras, y en 
particular las "de aventura". En la actualidad, la negativa del etnógrafo a adherir formalmente a un grupo de entrenamiento y abandonar la práctica del entrenamiento solitario, sigue produciendo escenas de campo valiosas. Pero sobre todo permite fluir a pleno la seducción etnográfica (Robben, 1995) por parte de los nativos, que no renuncian a cooptar al etnógrafo. Esas invitaciones "a entrenar juntos" se extienden a otros espacios de sociabilidad que en el caso de uno de los running teams se expande a encuentros sociales cotidianos de una amplia participación.

\section{Metodología}

\section{Definiciones etnográficas}

Para poder estudiar un estilo de vida como el running es necesario diseñar estrategias metodológicas precisas que conjuguen herramientas y estrategias de investigación complejas y que permitan llevar al máximo las posibilidades de conocimiento antropológico. Una etnografía de un fenómeno principalmente urbano y globalizado como el running demanda ocuparse de contextos de investigación amplios, cambiantes, que se reconfiguran a medida que se avanza en la investigación en el terreno. Por eso es que se diseñó un trabajo de campo multisituado (Marcus, 1995; Hannerz, 2003), característico de las etnografías "en casa" (Reed-Danahay, 1997; Gil, 2002; 2006), dado que se trata de un terreno que, como construcción espacio-temporal, suele estar en movimiento y posee un marcado carácter virtual. Por supuesto, ese diseño exige continuos reajustes e incorporación de otras estrategias situadas, ya que son las circunstancias las que definen el método y no al revés (Amit, 2000). Ello obliga a formular una estrategia metodológica sostenida en la "frecuentación profunda" (Clifford, 1999), entendida como "un conjunto de prácticas de investigación corporizadas, de pautas de separación, de distancia profesional, de ir y venir" (Clifford, 1999, p. 118). Durante más de tres años se han llevado intensas labores etnográficas que abarcan un amplio número de actividades, como participaciones en carreras en diversos lugares del país, observación de las competencias organizadas en la ciudad de Mar del Plata, además de sesiones de entrenamiento de running teams (Gil, 2020). Precisamente, una parte sustancial del trabajo de campo se concreta realizando la misma actividad que los sujetos de estudio: corriendo. Es en las carreras, pero sobre todo en los entrenamientos (la mayor parte de ellos solitarios), en donde el etnógrafo no sólo experimenta el mismo esfuerzo físico de los corredores sino que se expone a las situaciones cotidianas de quienes abrazan la pasión por correr como un estilo de vida (Gil, 2018). En ese sentido, se han compartido entrenamientos con diversos corredores, muchas de cuyas interacciones se produjeron de manera casual durante sesiones de carrera solitaria en las que se atreviesan las zonas más transitadas por los corredores en Mar del Plata, en su franja costera. En el marco de la participación de algunas de esas carreras se producen viajes grupales, en muchos casos en los vehículos de los corredores pero también, para las competencias más importantes, en buses especialmente contratados. Esos viajes constituyen experiencias fundamentales en las que se pueden incorporar densos datos etnográficos de la carne y la sangre de la vida cotidiana de los runners. Esos traslados que pueden llegar a durar casi una semana han permitido una intensa convivencia (por ejemplo compartiendo habitaciones en los hostels) con corredores de diversos running teams, muchos de los cuales suelen conocerse por compartir las continuas carreras que se organizan en la ciudad de Mar del Plata y sus márgenes. Del mismo modo, la participación en carreras por etapas permitió la convivencia en campamentos con otros corredores antes desconocidos durante tres días, además de los eventuales trayectos compartidos en pruebas de más de diez horas. En muchos de esos casos se establecen relaciones durables gracias a las redes sociales con eventuales reencuentros en otras competencias en el trascurso de los años.

Independientemente de este artículo, esta etnografía multi-situada contempla además el análisis de revistas especializadas, libros, diarios de circulación masiva, emisiones televisivas y cinematográficas, campañas 
publicitarias en medios audiovisuales, programas radiales, páginas webs, blogs, perfiles y biografías en las redes sociales. Todos ellos constituyen un material empírico que una investigación antropológica de nuestra contemporaneidad difícilmente pueda descartar. Además se desarrolla una antropología digital "remite al estudio etnográfico de las culturas digitales, pero también se refiere al desarrollo y la aplicación de metodologías digitales para mejorar la investigación etnográfica" (Born \& Haworth, 2017: 70). Por último, se apela a una etnografía autobiográfica (Gil, 2002) que permite utilizar episodios de un pasado que involucra un paso por el atletismo infantil, una costumbre nunca abandonada de salir a correr desde la adolescencia y el paulatino involucramiento en las carreras del colectivo runner desde 2005 a partir del desafío (Hanold, 2016) de participar en un medio maratón (21 kilómetros). Esta apelación a la reflexividad no implica una excusa para la autorreferencialidad, sino que por el contrario se utilizan fragmentos autobiográficos que aportan no sólo fuentes heurísticas sino también datos etnográficos de relevancia. De esa manera, se busca aprovechar productivamente los recuerdos de más de tres décadas de salir a correr, lapso en el que no sólo se modificó sustancialmente la denominación de la actividad sino que los hábitos, imaginarios, valores que acompañan el ejercicio de correr (antes jogging) -o trotar como le siguen llamando algunos incautos desactualizadosexperimentaron transformaciones de relieve.

\section{Apunes autoetnográficos}

Durante años, el crecimiento exponencial de personas corriendo en la costa marplatense o en los Rosedales de Palermo, donde eventualmente también entrenaba con Mariano, mi amigo de la infancia, me resultó algo notorio. A ese aumento de practicantes se le fue sumando la cada vez mayor legitimidad y prestigio que iba adquiriendo la pasión por correr, así como otros fenómenos que se iban suscitando, como la llamativa estilización de la práctica o la aparición del grupos de entrenamiento para aficionados, los running teams. Como eventual corredor solitario estas nuevas agrupaciones me resultaron extravagantes, sobre todo en comparación con los clubes de atletismo que frecuentaba a mediados de la década de 1980. De manera paulatina, fui mirando el fenómeno con mayor actitud antropológica, abordando incluso de manera reflexiva mis prejuicios hacia esas dimensiones "frívolas" y a la moda de esta nueva pasión masiva y mundializada. Con frecuencia apelaba en mis clases a ejemplos etnográficos del running, procedentes de mis observaciones mientras entrenaba y tomaba parte de algunas competencias, lo cual fue esporádico durante bastante tiempo. En un principio, había tomado el medio maratón de Buenos Aires de 2005 como un desafio personal, alimentado por el compromiso de amistad con Mariano para compartir la carrera. Así es que pude correrlo ese año y también en 2008, ya algo mejor entrenado y con la experiencia de saber que podía completar una distancia que en aquel momento me parecía un esfuerzo descomunal. Los tres años que transcurrieron entre una experiencia y otra (mi amigo sí corrió las ediciones 2006 y 2007) me mostraron una serie de cambios cualitativos de relieve en la pasión por correr. La multinacional Adidas ya se había hecho cargo de la organización y había implementado algo que hoy es prácticamente una condición sine qua non: el kit del corredor. De aquella remera blanca de algodón, y de una organización relativamente precaria en infraestructura y logística se había pasado al montaje de una expo maratón, a la contratación de animadores y la participación de figuras del espectáculo, incuso algunos de ellos como participantes. Aunque las redes sociales estaban en estado incipiente, ya se estaban cristalizando las estrategias de presentación personal de una pasión que estaba también siendo adoptada por famosos que se subían al fenómeno, algunos de ellos presentándolo como el camino hacia una vida mejor.

Durante más de una década transcurrieron varios períodos discontinuos de entrenamientos y la idea siempre vigente (y compartida con mi amigo) de correr algún maratón, al menos uno, antes de que seamos demasiado viejos como para ya ni poder intentarlo. De algún modo confiábamos en el slogan que difunden organizadores de carreras por las redes sociales: "El corredor que empieza una maratón no es el mismo que la termina". Todavía en nuestros treinta, parecía haber tiempo, pero las obligaciones familiares, 
personales y laborales (y eventualmente alguna lesión) muchas veces complicaban la posibilidad de mantener una continuidad y nivel de entrenamiento aceptable como para pensar seriamente en esos desafios. Todo cambió cuando la promoción de una carrera en tres etapas con campamentos intermedios me movilizó para intensificar y sistematizar algo más los entrenamientos. Aquel Raid de los Andes, con etapas de $25 \mathrm{~km}$ en Salta (de Chorrillos a Campo Quijano), $21 \mathrm{~km}$ en Jujuy (de Tumbaya a Purmamarca) y $11 \mathrm{~km}$ en las Salinas Grandes (provincia de Jujuy), me obligó a llevar una disciplina de entrenamiento desacostumbrada que hizo tambalear las convicciones de seguir inscribiéndome en ese tipo de carreras. Una semana antes de participar, con entrenamientos de más de $20 \mathrm{~km}$ iniciados antes de que salga el sol en dos maravillosas mañanas de marzo en Mar del Plata en un fin de semana, me llevaron a una sola conclusión: "hay cosas que se hacen sólo una vez en la vida". Suponía, sin la menor duda, que la férrea disciplina desplegada y las privaciones de las últimas semanas estaban justificadas por única vez ante la posibilidad de correr por los senderos del "Tren a las Nubes", por los caminos montañosos que desembocan en Purmamarca con una vista privilegiada del famoso "Cerro de los siete colores" y la experiencia de correr en una superficie de sal a una altitud imposible (las Salinas Grandes, a 4 mil metros sobre el nivel del mar). Menos de año después ya había participado en una carrera, el Cruce (de los Andes), a la que había clasificado como imposible para mis posibilidades y disposición para entrenar. Esta carrera es una competencia de alrededor 100 kilómetros en montaña en la frontera entre Argentina y Chile en tres etapas diarias de recorridos similares. ${ }^{3}$ Pocos meses antes de concretar El Cruce, también había completado mi primer maratón, en la ciudad de Buenos Aires. Tres años después -con más de un año de descanso obligado por una severa lesión en el tendón de Aquiles- me entrenaba para mi primera ultra maratón de 70 kilómetros en San Martín de los Andes.

\section{La experiencia encarnada del corredor}

Al menos desde Wacquant (2006), la utilización del cuerpo del propio investigador como un instrumento metodológico para llevar adelante etnografías de prácticas deportivas se ha transformado en todo un lugar común. Por supuesto, el foco en el cuerpo del antropólogo como una forma posible e incluso necesaria de conocimiento tiene una tradición mucho más densa (Blacking, 1977) que hasta podría remontarse a un clásico del pensamiento social como Marcel Mauss. Como señalan Lasalle, Recours \& Griffet (2019), la actividad de correr envuelve un amplio número de significados y técnicas diferenciales según la disciplina, la actividad, el terreno o la distancia que se debe recorrer. Pero también refiere a una apropiación diferencial según las edades o el género, entre otras posibilidades. En efecto, correr puede remitir a una manera de desplazarse velozmente en solitario o en grupo, a una exigencia de los límites del propio cuerpo, a un divertimento o hasta un sufrimiento, además de un mero recurso para quemar calorías. En ese sentido, estamos atravesados ya desde hace tiempo por un contexto global en el que un gran número de personas "cada vez más sienten la ineludible necesidad de «hacer algo con su cuerpo», movilizándolo más allá de los hábitos corporales que les imponen sus vidas cotidianas" (Ascheri \& Citro, 2012, p. 9). Ya desde el concepto de técnicas corporales de Marcel Mauss (1991), la antropología no ha cesado (con mayor o menor énfasis) de destacar la importancia de la corporalidad como un instrumento privilegiado a partir del cual comprender cómo se producen los procesos culturales. En efecto, los distintos colectivos construyen sus particulares modos de percepción, gestualidades y expresiones corporales, ya sea en las dimensiones cotidianas o rituales. Por ello es que el cuerpo "es el presente-ausente, al mismo tiempo pivote de la inserción del hombre en el tejido del mundo y soporte sine qua non de todas las prácticas sociales; sólo existe, para la conciencia del sujeto, en los momentos en que deja de cumplir con las funciones habituales, cuando desparece la rutina de la vida cotidiana o cuando se rompe «el silencio de los órganos»" (Le Breton, 1995, p. 124). Pero aquí no sólo se postula la importancia de la corporalidad y de las emociones, sino que se puntualiza en la relevancia que tiene el cuerpo del etnógrafo en el proceso de investigación. En 
consecuencia, resulta apropiado apelar a la perspectiva del embodiment (Csordas, 1990), que remite a un campo metodológico indeterminado que se define por la experiencia perceptual y por el modo en que el cuerpo se hace presente y se compromete en el mundo. Esta fenomenología cultural permite, entonces, entender el estar en el mundo, y su paso más avanzado debe conducir al reconocimiento de la mente en el cuerpo. Este aporte fenomenológico puntualiza en la importancia de aprender desde el cuerpo, de cómo el mundo se nos aparece a nuestras conciencias cuando alcanzamos un compromiso corporal. De esta forma, el embodiment es antes que nada una predisposición metodológica que se centra en la experiencia corporal, dado que el cuerpo es la base existencial del sujeto, pero también de la cultura (Csordas, 1999). En consecuencia, cobran preeminencia las dimensiones emocionales y simbólicas de la acción humana, así como las interacciones sociales, los controles institucionales, las transformaciones personales y también las interfaces entre lo humano y lo tecnológico. Por todo ello, estas herramientas conceptuales de la fenomenología implican un aporte significativo para describir e interpretar la experiencia humana y la forma en que se manifiestan los objetos de conciencia del "cuerpo vivido" y la "situabilidad" social (Crossley, 2001, p. 324) de esa conciencia encarnada.

En el caso que nos ocupa, los corredores desarrollan sus propios esquemas corporales (Crossley, 2001) que conllevan un conocimiento incorporado a modo de saberes de experto. Se trata de hábitos encarnados que le permiten a cada runner sobrellevar mejor cada acción física, y lo hace mejor a medida que acumula experiencias y lograr reflexionar sobre ellas. Esos esquemas corporales contemplan además un repertorio de disposiciones que permiten resolver desafíos puntuales inesperados, como terrenos sobre los que nunca se corrió. Por ejemplo, en el mencionado Raid de los Andes, el último trayecto se desarrolló a más de 4 mil metros sobre nivel del mar en las Salinas Grandes en la provincia de Jujuy. Pero allí no acabó la novedad de la experiencia inédita para la casi totalidad de los corredores, ya que los 11 kilómetros programados debieron transitarse con partes cubiertas por más de 20 centímetros de agua. Cuando el director de la prueba (un experimentado ex-atleta de alto rendimiento) se le preguntó por el modo de correr en esas condiciones se limitó a contestar "no tengo la menor idea". En efecto, esa etapa se caracterizó por la incertidumbre de correr con tan poco oxígeno y además no tener la posibilidad de apreciar con claridad un terreno repleto de pequeños pozos. Lo mismo vale para situaciones en las que se debe improvisar, desde cambiar el ritmo para superar a otro competidor o disminuirlo antes la exigencia inesperada del trayecto. En efecto, estas disposiciones tienen la capacidad de moldear la percepción, el pensamiento y la acción, en el marco de una flexibilidad y experticia que se va construyendo a medida que se incorporan hábitos a partir de la propia experiencia o el consejo de un entrenador (por ejemplo, cambiar la forma de la pisada). Todo ello constituye un proceso encadenado de acción sobre acción (Howe \& Morris, 2012). Así es que entre los tantos hábitos "incorrectos" de los corredores puede destacarse una forma de correr que se conoce como "talonear". Se asume que esta modalidad de carrera no sólo desgasta más al corredor sino que es más propensa a generar lesiones, sobre todo en el tendón de Aquiles. Pero cuando el corredor se decide a hacer el cambio, debe realizar un gran esfuerzo para encarnar los nuevos movimientos. El propio etnógrafo atravesó ese proceso y aún no ha encarnado completamente la manera de pisar con la punta del pie para evitar sobrecargar los talones y sus maltrechos tendones de Aquiles. No es otra cosa que ese "cuerpo recesivo" (Allen-Collinson \& Owton, 2015), que está en gran parte ausente del pensamiento consciente en la vida cotidiana, en términos del funcionamiento general y mundano de los órganos internos y procesos fisiológicos tan elementales como la respiración. Sin embargo, este cuerpo "ausente" se lleva a una mayor conciencia cuando el dolor o la enfermedad, o incluso el intenso placer, nos recuerdan su presencia. Luego, "el cuerpo 'desaparece' y es llevado, a veces de manera aguda, a la conciencia" (Allen-Collinson \& Owton, 2015, p. 247). 


\section{RESULTADOS Y DISCUSióN}

\section{Sentidos y corporalidad}

Entre las dimensiones sensoriales que intervienen en la experiencia de correr, la dimensión háptica cobra una notable relevancia. Habitualmente reducido a lo táctil, lo "háptico" se extiende más allá del contacto cutáneo directo, para incluir sensaciones corporales sentidas internamente. Lo háptico, para Paterson (2009), incluye la cinestesia (sentido del movimiento), la propiocepción (sentido de la posición de los msculos) y el sistema vestibular (sentido del equilibrio). La acción de correr, y mucho más hacerlo de modo sistemático y apasionado, coloca al cuerpo de frente a una amplísima variedad de experiencias y sensaciones y a una vinculación estrecha con los fenómenos más ordinarios de la naturaleza. Así, nos vamos exponiendo de modo ocasional al sol abrasador, al viento que corta o impulsa la carrera, a una suave brisa que reconforta, a una fuerte lluvia que moja y torna resbaladiza la superficie o hasta la nieve en algunos lugares fríos y de montaña. Noches gélidas, días soleados, jornadas lluviosas, vientos helados que perforan los pulmones configuran un men de situaciones posibles que el corredor puede seleccionar de forma relativa (en los entrenamientos, pero no tanto en las competencias) ante los márgenes que le ofrece el clima. En efecto, cada corredor suele conocer sus puntos débiles, como posibles dificultades para correr con temperaturas elevadas. Ello obliga a ese tipo de corredores, por ejemplo en temporada estival, a postergar sus entrenamientos para la noche o hacerlo por la maana muy temprano, lo que requiere una considerable autodisciplina. El cuerpo deshidratado no sólo disminuye el rendimiento sino que implica un riesgo de salud manifiesto. Así es que, por ejemplo, el propio etnógrafo intenta evitar al máximo los días de fuerte viento, lo cual durante ciertas épocas es casi imposible en una ciudad como Mar del Plata. En definitiva, son todas experiencias de calentamiento y termorregulación (Allen-Collinson \& Owton, 2015), que se producen por el propio cuerpo y también con la ayuda de la indumentaria. La estrategia de vestimenta no es un aspecto superficial, ya que la temperatura del cuerpo cobra relevancia para cualquier deportista. Sobre todo a cierta edad, tendones y msculos "fríos" son territorio ideal para lesiones, por lo que es necesario llevar al cuerpo a una temperatura y flexbilidad que prevengan sorpresas desagradables después. Incluso para las carreras, el calentamiento precompetitivo es para muchos corredores todo un ritual detallado y al que se le presta especial atención. Las carreras masivas se caracterizan por un ir y venir interminable de corredores solitarios y en grupo poniendo al punto justo de temperatura a su cuerpo y ajustando la flexibilidad muscular. Porque además, "el calentamiento incorpora un elemento energizante, por lo que una sensación de calor también se puede experimentar como sentimiento de energía interna y preparación corporal" (Allen-Collinson \& Owton, 2015, p. 256).

Algunas carreras, como los ultramaratones, obligan a colocar un cuidado extra en la termoreregulación del corredor. La extensión de la carrera, que puede demandar entre 7 y 24 horas (o incluso más en las de 100 millas), los horarios en los que se corre (muchas largan de madrugada con temperatura bajo cero), las diferencias de altitud y otro conjunto eventual de posibilidades geográficas, constituyen verdaderos desafíos de estrategia y resistencia para el corredor. Algunas de estas competencias, las de mayor logística y organización, le ofrecen al corredor la posibilidad de cambiar su ropa y hasta dejar prendas en los puestos de asistencia. En esas pruebas, se llegan a experimentar variaciones térmicas de más de 20 grados durante todo el trayecto, con una eventual largada usando guantes, gorro, calzas largas, remera "primera piel" y un polar, para luego terminar corriendo sólo con la remera técnica de la carrera y un pantalón corto. Estos ejemplos permiten visualizar que dimensiones como el tacto, la textura, el movimiento y la temperatura atraviesan la experiencia del cuerpo vivido, en las que, no está de más reiterarlo, esa dimensión "ornamental" (la indumentaria) es parte fundamental de la experiencia de correr. Por ejemplo, las medias mojadas, si no se toman recaudos previos (como pasarse vaselina en los pies antes de la carrera) pueden destrozar los pies del corredor. Muchas carreras atraviesan ríos, arroyos, y lodazales que desafían cualquier estrategia ingenua. A veces, los alertas de un desgaste cutáneo peligroso no llegan a tiempo y cuando el dolor se impone puede tratarse de una 
molestia capaz de complicar la continuidad en una carrera o la participación al día siguiente cuando se trata de competencias de etapas sucesivas. Cuando más dilatada sea la experiencia mayor será la "agudeza háptica" (Allen-Collinson \& Hockey, 2011) del corredor en sus prácticas deportivas encarnadas.

La densidad, riqueza, complejidad (y hasta ambigüedad) de la fenomenología del corredor nos enfrenta a configuraciones emocionales características que refieren a experiencias personales, colectivas y hasta de interfaz con el medio ambiente (cargarse de energía mientras se escala una montaña o se atraviesa un río en una carrera de trail running ${ }^{4}$ ) y con la tecnología (controlar nuestro ritmo y distancia con un reloj GPS o correr escuchando música) ${ }^{5}$. Además de las emociones (euforia existencial, plenitud, satisfacción personal) que caracterizan el colectivo runner, otro tipo de situaciones menos "glamorosas" invaden de manera continua la experiencia de correr, como el cansancio, la presión y, sobre todo, las lesiones. Sentimientos como el dolor o la fatiga (corporal y mental) además suelen traducirse en emociones (como la frustración) que drenan la energía de los corredores que pueden hasta replantearse la continuidad apasionada en este estilo de vida. Pero sobre todo, las lesiones constituyen uno de esos sucesos claves que pueden amenazar la viabilidad de un itinerario runner durable y, en gran medida, constante. Es lo que le ocurre al propio etnógrafo, que desde 2012 arrastra una tendinosis crónica en el tendón de Aquiles de la pierna izquierda, además de otro tipo de molestias y afecciones menos graves (una pubalgia, tirones, sobrecargas) que se han ido experimentando con el entrenamiento y la ayuda del paso de los años. Durante muchos lapsos, algunos bastante largos, mi (relativa) baja forma física limitó de modo considerable mis posibilidades de avances en el terreno y de acceso a experiencias etnográficas claves. En efecto, no disponer de un estado físico acorde a la exigencia de los corredores "serios" impide seguir el ritmo de muchos interlocutores en los entrenamientos, lo que lleva directamente a suprimir esa posibilidad o al menos a considerarla en menor medida. Una buena parte de los corredores serios, algunos aficionados con exigencias similares a un atleta de elite, llevan adelante entrenamientos, frecuencias y ritmos de carrera imposibles de equiparar para las posibilidades de un etnógrafo que no adhiere a estándares tan rigurosos. Ello es muy evidente cuando se busca interaccionar con los runners más apolíneos (Gil, 2020), que poseen una rigurosa disciplina de entrenamiento, con fuertes ritmos de carrera y horarios estrictos. Pero la posibilidad de evidenciar cierta actitud y aptitud para correr (en entrenamientos y en carreras) contribuye a obtener cierto respeto y cercanía con los sujetos de estudio. Así es que el cumplimiento de performances aceptables y la demostración de conocimiento y experiencia (sobre todo en el género de trail running que requiere saberes específicos inimaginables para un corredor urbano) colocan al etnógrafo en otra dimensión. Por eso, esos momentos de baja forma física también hicieron perder cercanía con los sujetos de estudio, en gran parte por no estar condiciones de inscribirme en carreras, al menos en las de 10 kilómetros.

Más allá de que el running es central en mi vida por haberlo construido como mi tema de investigación, la continuidad en el entrenamiento nunca pudo ocupar una posición central. Sólo la obligación de inscribirme con anticipación a alguna carrera fue una estrategia algo eficiente para entrenar con más regularidad. Digo relativamente porque a pesar de que pude cumplir con esos desafíos, nunca llegué con el entrenamiento adecuado, aunque se trata de una sensación que aqueja a muchos corredores "serios" con mayor disciplina que este nativo marginal. Así es que, por ejemplo, en una carrera en Laguna de los Padres en 2019 apenas si pude completar los $15 \mathrm{~K}$, tardando diez minutos más que el año anterior, cuando corrí relajado junto con Guille y Cachito, conversando y bromeando y hasta posando para algunas fotos de la organización. Habían sido semanas complicadas, recuperándome de una gripe y sufriendo fuertes dolores de dos lesiones severas de mi adolescencia (muñeca y rodilla derechas) que ya creía controladas. La semana previa apenas si pude trotar y temí no poder correr el día de la competencia, pero entre la adrenalina y el ibuprofeno pude completar una de las carreras más bellas del circuito "de aventura" de Mar del Plata y la zona. Por lo general, las decisiones de participar en alguna competencia con poco entrenamiento se debió al compromiso de hacer trabajo de campo y retomar contacto con mis interlocutores. Además, las obligaciones (laborales, familiares) y vicisitudes (lesiones, enfermedades) confluyen para discontinuar los entrenamientos, sobre todo para un 
etnógrafo que carece de los compromisos emocionales de los nativos, como la vinculación a un grupo de entrenamiento. A tal punto que mi rutina de fútbol semanal que lleva dos décadas (aunque también con sus intermitencias por lesiones o por cercanía de alguna carrera exigente) no deja de provocar incredulidad y rechazo de los corredores por seguir manteniendo ese hábito al que consideran contraproducente y propenso a generar lesiones. En efecto, ambas actividades me generan una retroalimentación negativa a mis 47 años, perjudicándome notoriamente en mi desempeño y hasta en mi condición física. Cuando obtengo un buen entrenamiento de fondo (largas distancias), mi cuerpo se muestra más lento y torpe para las destrezas futbolísticas. Y además, un esfuerzo importante en el fútbol semanal de los martes me produce una sobrecarga muscular y agrega molestias en los tendones de Aquiles que suelen complicar mis posibilidades de entrenamiento en los días posteriores.

\section{Carreras y energía emocional}

El running como estilo de vida contiene un ritual sustancial que se constituye en el eslabón fundamental de las densas cadenas de interacción de los corredores: las carreras (Gil, 2019). Según Shipway, Holloway \& Jones (2013), estas pruebas resultan claves para moldear las normas subculturales y las identidades en el running. En la misma línea, afirman que "la participación en carreras de larga distancia es una oportunidad para la autorrealización y la autoexpresión, que son beneficios que no siempre se encuentran en la vida cotidiana" (Shipway et al., 2013, p. 273). La utilización del clásico concepto de subcultura presenta el problema de entender estos colectivos como internamente homogéneos en cuanto a valores y representaciones colectivas. Más allá de emplear o no esa categoría, "un modelo de subcultura que se basa en el significado compartido de una red de grupos, expresado en la acción, permite una comprensión de la relación entre el grupo y la comunidad en general en la que el grupo está integrado" (Fine, 2012, p. 144). Así es que estos sucesivos rituales de interacción (Collins 2004), que pueden implicar además un viaje previo de miles de kilómetros, producen en los corredores esa energía emocional que le otorga la robustez que caracteriza al universo del running en nuestra contemporaneidad.

Las emociones pueden concebirse como "la energía interna que nos impulsa en un acto, lo que da cierto «carácter» o «colorido» a un acto" (Illouz 2007,p. 15). Al cargar de energía nuestras acciones, las emociones suponen "al mismo tiempo cognición, afecto, evaluación, motivación y el cuerpo. Lejos de ser presociales o preculturales, las emociones son significados culturales y relaciones sociales fusionados de manera inseparable, y es esa fusión lo que les confiere la capacidad de impartir energía a la acción” (Illouz 2007, p. 15). En definitiva, "las emociones son aspectos profundamente internalizados e irreflexivos de la acción, pero no porque no conlleven suficiente cultura y sociedad, sino porque tienen demasiado de ambas" (Illouz 2007, p. 16). La energía emocional que se genera en las carreras opera, en la racionalidad de los corredores, como una poderosa fuerza impulsora, en este caso para entrenar e inscribirse en carreras de forma regular. A través de las redes sociales es en donde los corredores se expresan más elocuentemente acerca de sus sentimientos y emociones. En una de sus detalladas crónicas y relatos en Facebook, Juan destacó en una oportunidad mientras se preparaba para una ultramaratón en la selva misionera: "Desafío a mí mismo; a mis miedos e inseguridades. Es la búsqueda de la superación misma, es vencer el dolor, la desazón, el desánimo y los imprevistos. Es alimentar esa fuerza interior que te impulsa hacia adelante día a día, es la búsqueda de los sueños". De la eficacia que tengan esas carreras para producir emociones positivas dependerá también la posibilidad de crear y recrear los símbolos de la pertenencia grupal. Incluso en sus rendimientos destacados, muchos corredores optan por destacar la relevancia de pertenecer a un running team. No sólo por los compañeros que "hacen el aguante" alentando a los que corren sino por la fuerza vital que se le asigna al grupo al que pertenecen.

Una carrera "exitosa" despierta entre los corredores deseos públicos de realizar nuevas apuestas que garanticen una continuidad en este estilo de vida. Las referencias que se obtienen en el campo giran en 
torno a sentimientos de alegría, orgullo, confianza, fuerza, solidaridad con otros corredores (en particular cuando se pertenece a un grupo de entrenamiento), satisfacción plena. Tanto en las redes sociales como en las conversaciones cotidianas, las impresiones "positivas" luego de una carrera afloran a partir de discursos autocelebratorios de la identidad individual y grupal (los runners en general, los miembros de un runningteam en particular). Se configura un "contagio emocional" en todas las "situaciones" que hacen fluir palabras, gestos, y ciertas imposturas que dan cuenta de cómo se potencia la solidaridad grupal. Algunos apuntes de campo de la última carrera en la que participé durante 2019 en Pinamar, puede ilustrar la intensidad de estos rituales de interacción y de la experiencia corporal y emocional del etnógrafo. El circuito que se monta cada año en esa ciudad bonaerense es sencillamente hermoso y se atraviesan lugares inimaginables para quienes incluso vivimos en zonas costeras. La inmensidad de la franja de arena en el límite norte de esta ciudad turística, los desafiantes médanos y la interminable fila de corredores transitando esos senderos son experiencias imposibles de repetir en otros contextos. Lo mismo para el ingreso en las zonas boscosas y desconocidas para el gran público, que configuran un trayecto discontinuo que desafía cualquier entrenamiento previo en superficies duras. En definitiva, casi 30 kilómetros desgastantes que llegan a desmoralizar a cualquier corredor pero que luego se transforman en recuerdos imborrables que derivan en el orgullo de haber podido completar un trayecto caracterizado por momentos de intenso dolor. Es en esos instantes cuando aparecen de manera súbita deseos irrefrenables de abandonar y terminar con el "sufrimiento". Paradojalmente son los momentos de mayor intensidad y disfrute, sobre todo cuando se los logra superar y los instantes de debilidad desaparecen para hacer fluir la energía emocional en toda su plenitud. Desaparecido aquel "infierno" de arena blanda y médanos empinados, superados los senderos boscosos repletos de raíces sobresalidas sobre un terreno blando y poceado, la aparición de las primeras áreas urbanas y un camino más estable devuelven el espíritu y el corredor recupera un ritmo de carrera que le permite soñar con un final próximo, aunque todavía queden casi 10 kilómetros. Así es que la parte final fue pleno disfrute, con una superficie dura y continua que atravesaba el campo de golf, una ancha avenida que conducía hacia la costa (Avenida Martín Pescador) y la llegada por la costanera (Avenida del Mar) sobre la céntrica Avenida Bunge, alzando a mi hija de 19 meses en los últimos metros del recorrido. Y, por supuesto, una vez atravesada la línea final, el reencuentro pleno y completo con la familia que soportó las casi tres horas de ausencia.

Por el contrario, como indica Collins (2005) para los rituales fallidos, una carrera mal organizada, una performance decepcionante sin que medien atenuantes, un circuito poco placentero o cualquier otra fuente potencial de malestar (por ejemplo, incompatibilidad con los compañeros del grupo de entrenamiento), drenan la energía emocional de los corredores. Por supuesto, muchos runners experimentados pueden sufrir decepciones que no consiguen desanimarlos. Como también expresó Juan en Facebook, luego de abandonar en un ultramaratón: "una de las experiencias mas lindas de mi vida como corredor, el trago amargo, no poder terminarla... el consuelo de tonto: de los 1020 inscriptos habrán finalizado algo así como 150. Lo bueno: habrá que regresar por la revancha". Precisamente, la idea de "revancha", de repetir una carrera frustrante para revertir el sinsabor de un rendimiento decepcionante, impide que se drene la energía emocional en esos casos. Esta clase de situaciones muestran que aunque se podría plantear que correr o visitar un lugar para correr (y eventualmente no volver más) es lo contrario que habitarlo, los corredores desarrollan profundos arraigos con esos lugares. De hecho, mucho runners sienten (como el propio etnógrafo) que dejaron algo suyo en alguna montaña, en un sitio en particular o en todo el conjunto de la carrera. De allí que no pocos corredores sueñen con retornar a los mismos lugares, como corredores o como visitantes, para volver a encarnarse con un entorno que tanto significa, por lo que se logró o por lo que no se pudo conseguir. Porque correr es una actividad que configura temporalidades y espacialidades distintivas que pueden remitir a un tránsito fugaz y transitorio, pero también a lo duradero.

En esa misma dirección, al releer las notas de campo se advierte de qué manera impacta una mala carrera en la energía emocional de cualquier corredor, incluso en la de un nativo marginal como el etnógrafo, para quien el running no implica una opción de estilo de vida ni una referencia identitaria. En algunas carreras se 
disparan sensaciones diferentes y ambiguas. Así es que se puede estar frustrado por el bajo rendimiento pero ese episodio tal vez tenga la capacidad de favorecer una nueva carga de energía emocional a modo de desafío para correr mejor la próxima edición u otra carrera programada. Los sentimientos de cansancio, incluso debilidad, bronca y frustración pueden ser insumos movilizadores que permiten un análisis retrospectivo de los errores en la carrera o en el proceso de entrenamiento. Sobre todo las carreras de trail suelen enfrentar a los corredores no muy experimentados a graves errores de estrategia que son enseñanzas -si se las sabe procesarpara otros eventos. En ese sentido, se ha podido registrar que algunos entrenadores y corredores transmiten ideas poco realistas del ritmo de carrera que se debe llevar para un aficionado en terrenos con grandes desniveles y grados de dificultad. De todos modos, a partir de esas experiencias, será posible reformular los objetivos y analizar reflexivamente el futuro. En el mejor de los casos, aunque no se haya alcanzado el rendimiento programado, cuando la efervescencia embarga al corredor, se impone la pulsión por emprender nuevos desafíos y eventualmente mejorar el entrenamiento para alcanzar performances más destacadas o planificar una carrera en mejores condiciones o "llegar más entero" al final de una competencia. En mis carreras, los rendimientos por debajo de las expectativas nunca despertaron las mismas emociones. En algunos casos la frustración drenó la energía emocional a tal punto de desembocar en períodos inmediatos de poco entrenamiento y alejamiento de las carreras. En otras ocasiones, el cansancio extremo en la línea de llegada fue un aprendizaje para el siguiente objetivo, como me ocurrió en las dos ocasiones en la participé en carreras por etapas. En esas dos competencias, las primeras etapas fueron sumamente desgastantes y me hicieron dudar acerca de las posibilidades de completar los dos tramos exigentes que quedaban. Sin embargo, las estrategias más conservadoras en los días siguientes redundaron en mejores rendimientos y carreras más relajadas. Y sobre todo, fueron lecciones muy contundentes para respetar los terrenos irregulares y las montañas. Así aprendí a entrar en sintonía con el desgaste corporal para evitar sorpresas, desde calambres inesperados hasta un agotamiento imposible de controlar y recuperar.

De todos modos, más allá de los imponderables de la fenomenología de cada corredor, es en estas interacciones (algunas de ellas cotidianas como los entrenamientos y otras más extraordinarias como las carreras) en donde se experimenta la proximidad física y se alcanzan un estado de "intersubjetividad intensificada" (Collins 2004, p. 33), una retroalimentación constante que remite a la noción de efervescencia colectiva de Durkheim. Esas experiencias se pueden objetivar en determinados símbolos, como una remera de la carrera (y que luego se lucirá en los entrenamientos) o la medalla de finisher, que prueba la membrecía a esa comunidad moral de corredores. En definitiva, a partir de estas situaciones, de esos encuentros temporales y de esa copresencia de cuerpos, los corredores se cargan mutuamente de emociones por el efecto de esas cadenas de encuentros (carreras) pasados y presentes. Como lo captó en uno de sus publicidades en las redes sociales una empresa organizadora de eventos, "la línea de meta es sólo el comienzo de una nueva carrera". La energía emocional generada se constituye entonces en un impulso para recrear y hasta incrementar esas cadenas de interacción. Todo ello ocurre en el marco de una atención focal compartida en la que se produce una coordinación recíproca de emociones que tienden a perdurar en torno a una armonía que crea lazos identitarios.

Los discursos hegemónicos del running suelen insistir en motivos y lugares comunes que giran torno a la configuración de una moralidad runner que contiene una ética de la autosuperación que se construye a partir del esfuerzo constante y la superación de los obstáculos que se presenten, ya sean físicos o mentales. Esa actitud del corredor, que es todo un posicionamiento moral, debe representar un itinerario de endurecimiento emocional que le permitirá sobreponerse a potenciales adversidades. "Dolor", "esfuerzo", "trabajo duro", "educación", "voluntad", son todos términos que se conjugan para conformar una comunidad moral que además siembra el ejemplo a los no iniciados o a los novatos que aún no están convencidos por completo. Tales discursos muestran que "la capacidad de soportar el dolor" (Bridel, Markula \& Denison, 2016) es uno de los rasgos salientes de la identidad de los corredores (Bale, 2006; Shipway \& Holloway, 2016). En efecto, el placer y los límites del dolor se desdibujan de modo permanente. De hecho, el sufrimiento como 
"dolor positivo" (Howe, 2004) se experimenta "como un vehículo para la autoexploración y la trascendencia: como una técnica para 'forzar' los pensamientos cotidianos y simplemente 'dejarlos ir"' (Atkinson, 2010, p. 118). Las lecturas dominantes en nuestro medio suponen que esta revalorización del dolor no es otra cosa que una postulación normativa que valoriza los cuerpos fuertes, disciplinados y dóciles para adaptarse a las exigencias del mundo laboral y el mercado de consumo (Gelfman, 2017, Iragola Cairoli, 2019; Hijós, 2019). Sin embargo, y como argumenta Atkinson (2007) "pocas personas practican deportes recreativos con el propósito expreso de lastimarse física, emocional y psicológicamente" (Atkinson, 2007, p. 166). Ello no excluye que en torno a determinadas actividades deportivas se pueda conformar una "comunidad de dolor" (Atkinson, 2007), como los triatletas estudiados por este mismo autor, que también practican deportes de manera intensiva y están dispuestos a resistir el dolor y hasta se entrenan para ello. Para esos triatletas canadienses, asumirse como capaces de disfrutar ese sufrimiento no siempre garantiza que podrán soportar una carrera determinada, por lo que se manejan en contextos de incertidumbre y ansiedad en el marco de la experiencia fisiológica de estar "agotado" física y emocionalmente. De todos modos, el mismo autor no puede dejar de moralizar negativamente estas técnicas corporales basadas en "códigos espartanos" (Atkinson, 2007) a los que equipara con formas religiosas de ascetismo, auto-mortificación y auto-flagelación, a modo de castigos rituales. Las carreras de larga distancia, y en particular los maratones, plantean el desafío adicional del conocido muro, por tratarse de un momento en el que el corredor siente de manera repentina el esfuerzo del trayecto realizado cuando todavía falta una parte considerable del trayecto. Se trata de una barrera física y psicológica entre los 30 y 35 kilómetros que remite a un descenso brusco en la energía del corredor. ${ }^{6} \mathrm{Si}$ bien existen muchos corredores aficionados que afirman no experimentar este muro, se trata de una experiencia que puede llegar a desafiar todos los límites imaginables de sensaciones relacionadas con el dolor, la resistencia y el miedo. Inclusive el corredor puede descubrir en esos episodios tolerancias desconocidas, como también partes del cuerpo (músculos que duelen) cuya existencia desconocía. Las explicaciones médicas señalan que ello se produce cuando el cuerpo modifica el sistema mediante el cual obtiene energía. En determinado momento se deja de utilizar eficientemente las reservas de glucógeno que se almacenan en el hígado y en los músculos, razón por la cual se aconseja consumir un volumen considerable de hidratos de carbono los días previos a una carrera para extender al máximo posible la aparición del muro. Por supuesto, eso dependerá de cada corredor, su entrenamiento, el ritmo de carrera, la hidratación, el consumo de hidratos de carbono durante la carrera (frutas, gomas y geles energéticos) y el peso del corredor, entre otras variables.

\section{La conexión con la naturaleza}

Howe \& Morris (2009) han destacado la interrelación entre los cuerpos de los corredores con los entornos "naturales" que se habitan en entrenamientos y competencias. Siguiendo a Ingold, estos autores plantean que manejamos los paisajes a partir de una conciencia muscular que hace posible que el movimiento se encarne (Ingold, 2000; Howe \& Morris, 2009). Esos paisajes "de ocio" adquieren en principio un sentido visual y suelen estar separados espacial y temporalmente de los lugares del trabajo diario y las rutinas cotidianas. En esos espacios, habitualmente novedosos, el corredor recoge información utilizando todos sus sentidos, por lo que ese mundo que lo rodea adquiere su significado y propósito en un proceso continuo de coproducción entre los cuerpos que corren y el entorno que lo rodea. El corredor utiliza sus experiencias previas y se vincula con esos entornos de modo diferente, por ejemplo para recargar energías, como esas pendientes empinadas que se caminan a paso firme en una carrera de trail de larga distancia. Otros momentos pueden ser ideales en la fenomenología del corredor para conectarse con el paisaje y poner a nuestro cuerpo (y sus dolores, su cansancio) en suspenso. La naturaleza puede transformarse en un verdadero "santuario" (Howe \& Morris, 2009) que ofrece un respiro de paz, belleza y relajación, además de que permite otras exigencias (como las lomas, las diferencias de terreno) que favorecen la construcción de un cuerpo más duro y preparado para 
carreras exigentes. Esos paisajes sufren modificaciones constantes desde el momento en que son habitados por corredores (en entrenamientos y ocasionalmente en carreras) que dejan su marca indeleble. Entonces pueden aparecer rastros, como esas señales por donde transitar con más comodidad o, en el peor de los casos, los residuos típicos de la acción humana, como los distintos elementos de plástico que tanto dañan al ambiente (botellas, bolsas, envoltorios de geles energéticos, etc.). En efecto, más allá de una serie de comportamientos que preocupan a los organizadores de las carreras de trail debido al escaso cuidado del entorno (muchos corredores arrojan residuos de forma indiscriminada durante las carreras) lo que prima es un tipo de vinculación estrecha con la naturaleza. Ese conjunto de entidades no humanas (Ingold, 2000; Latour, 2008) forma parte de una compleja de red de relaciones que involucran, en la fenomenología del corredor, hasta intercambios energéticos indispensables para lidiar con los esfuerzos más exigentes. Los complejos y variados ejercicios que se realizan en este tipo de carreras (escalar y descender montañas, atravesar caminos de diversa complejidad y peligrosidad) se van incorporando a una experiencia corporal (Ingold, 2000) en la que los sentidos se experimentan como un todo. Porque la naturaleza no está simplemente "allí" para ser expuesta o descubierta por el corredor, sino que emerge activamente en una amplia variedad de formas a través de los hábitos, rutinas y prácticas asociadas con la experiencia de correr. Al habitar esos espacios a través de un ejercicio físico, los cuerpos y las naturalezas se producen simultáneamente, o son "coproducidos" (Howe \& Morris, 2009). Esos cuerpos transitan espacios "invadidos" por la vegetación, caminos que se inundan ante las lluvias continuas o senderos boscosos que sufren interrupciones abruptas de un alambrado que lo separa de un campo de producción agrícola. Y así es que también los corredores modifican constantemente esos paisajes, como cuando llegan con sus autos a sus zonas acostumbradas de encuentro, lo que también es evidente en los paisajes de la costa de Mar del Plata. $\mathrm{O}$ aquellos grupos de entrenamiento que organizan sesiones de fin de semana (en general los domingos) en entornos naturales para descansar de la cotidianidad urbana y así entrenar en paisajes diferentes. Esta clase de lugares, como la Laguna de Padres y la Sierra de los Padres (alejadas 20 y 25 kilómetros de la ciudad) pueden generar momentos liminales de ruptura, de oxigenación del cuerpo y de encuentro con el medio ambiente, con tiempos más relajados y estéticamente marcados.

Más allá de que en una carrera solitaria en conexión con el entorno puedan alcanzarse, como postulan algunos runners, los estados mentales asociados al yoga, también se acepta -como afirman algunas expresiones habituales en las redes y numerosos los consejos de entrenadores-, que "sólo un corredor de fondo sabe lo buena compañera que es la soledad". Esa analogía con el yoga, más allá de las evidentes diferencias de las técnicas corporales involucradas en cada disciplina, surge de las concepciones nativas de los distintos practicantes y expertos. Así es que se pueden destacar nociones tales como "meditar en movimiento" y la enseñanza de técnicas de "conocimientos corporales relacionados con «sentir», «escuchar» el propio cuerpo; para garantizar la relajación, la flexibilidad y el equilibrio" (Lewin \& Puglisi, 2012, p. 255). Allí es donde el yoga muestra espacios de contigüidad con el modo es que es concebido el running por muchos de sus aficionados y expertos más reconocidos. Otras dimensiones de la corporalidad que se asocian al yoga, son las "sensaciones físicas" (D'Angelo, 2016), relacionadas con la respiración, la fluidez de pensamientos, la atención o la concentración. En este último caso, "se trataría de una percepción no del mundo, sino del propio cuerpo. El cuerpo es a la vez sujeto y objeto. La paradoja aparente de la corporalidad reside en que el cuerpo es visible a la vez que vidente, el cuerpo objeto se superpone al cuerpo fenoménico" (D 'Angelo, 2016, p. 27). De manera también análoga, los practicantes de yoga pueden concebirlo como un conjunto de técnicas corporales tendientes a "mejorar o mantener su salud o estado físico" (D'Angelo, 2016, p. 29) lo que implicaría una apropiación más "instrumental".

\section{Conclusiones}

A lo largo de este artículo se ha intentado mostrar la relevancia que el cuerpo del investigador tiene para estudiar un estilo de vida deportivo como el running. Aunque una gran parte de lo postulado en este 
texto podría aplicarse a otras prácticas y estilos de vida, una etnografía sobre el running coloca al investigador ante un conjunto de posibilidades y exigencias distintivas. No se trata sólo de la adquisición de esquemas corporales sino de la necesidad de mantener una predisposición y una actualización constante de su estado atlético para poder realizar trabajo de campo en mejores condiciones. En ese sentido, el compromiso corporal del etnógrafo se ha mostrado fructífero en múltiples dimensiones. Así es que la posibilidad de entrenar y participar en carreras con los sujetos de estudios favorece de manera notoria las relaciones en el campo, sobre todo si el investigador puede además mostrar cierta expertise que lo coloque como un actor con conocimiento en el terreno. Por ello es que se ha enfatizado en las dimensiones hápticas de la experiencia encarnada de correr, pero también de las configuraciones emocionales y de las conexiones que nuestros cuerpos experimentan con el ambiente. Todo ello ha confluido en un artículo que pretende, al tomar diversos enfoques teóricos y una estrategia metodológica situada, sentar algunas de las bases para describir y comprender la fenomenología del corredor. Así es que se ha intentado mostrar el tipo de exigencias que afronta un runner en sus entrenamientos y carreras, las emociones que lo embargan y las maneras en que se conecta con entornos que van desde grandes urbes a paisajes desiertos que pueden ser territorializados por miles de corredores en una competencia. Y finalmente, la condición de nativo marginal, de alguien que corre y participa en carreras pero que no pertenece a ningún running team y no respeta una amplia variedad de preceptos del corredor "serio" (entrenamientos discontinuos, excesos en comidas), ha sido utilizada para forzar un extrañamiento que permita exotizar con mayor precisión esa experiencia encarnada de lo que implica adoptar al running como un estilo de vida. Dolores "positivos", exploraciones de los límites mentales y físicos, lesiones, conexión con el ambiente y los fenómenos de la naturaleza, estrategias de vestimenta y de uso de la tecnología, son todas complejas dimensiones del estar en el mundo de actores que no persiguen el sufrimiento ni se someten a rituales de autoflagelación. En definitiva, sólo se trata de runners que buscan a través de este conjunto de experiencias corporizadas, acceder a una vida mejor y más bella.

\section{REFERENCIAS BIBLIOGRÁFICAS}

Abbas, A. (2004). The embodiment of class, gender and age through leisure: a realist analysis of long distance running. Leisure Studies, 23 (2), 159-175.

Allen-Collinson, J. \& Hockey, J. (2011). Feeling the way: Notes toward a haptic phenomenology of distance running and scuba diving. International Review for the Sociology of Sport, 46 (3), 330-345.

Allen-Collinson, J. \& Owton, H. (2015). Intense Embodiment: Senses of Heat in Women's Running and Boxing. Body \& Society, 21(2), 245-268.

Amit, V. (2000). Introduction: constructing the field. In V. Amit (Ed.). Constructing the field. Ethnographic Fieldwork in the Contemporary World (pp. 1-18). London and New York: Routledge.

Atkinson, M. (2008). Triathlon, suffering and exciting significance. Leisure Studies, 27 (2), 165-180.

Atkinson, M. (2010). Fell running in post-sport territories. Qualitative Research in Sport and Exercise, 2, 109-132.

Bale, J. (2006). The place of pain in running. In S. Loland, B. Skirstad, y I. Waddington (Eds.). Pain and injury in sport: Social and ethical analysis (pp. 65-75). New York: Routledge.

Blacking, J. (1977) Towards an anthropology of the body. In J. Blacking (ed.). The Anthropology of the Body (pp. 1-28). London: Academic Press.

Born G \& Haworth, C. (2017). Mixing it. Digital Ethnography and Online Research Methods-A Tale of Two Global Digital Music Genres. In L. Hjorth; H. Horst; A. Galloway \& G. Bell (Eds). The Routledge Companion to Digital Ethnography (pp. 70-86). New York and London: Routledge.

Bridel, W.; Markula, P. \& Denison, J. (2016). Critical considerations of runners and running. In W. Bridel, P. Markula, y J. Denison (Eds.). Endurance Running. A socio-cultural examination (pp. 181-195). New York: Routldege.

Citro, S. \& Ascheri, P. (2012). Presentación. In S. Citro \& P. Ascheri (Eds.). Cuerpos en movimiento. Antropología de $y$ desde las danzas (pp. 9-13). Buenos Aires: Biblos. 
Clifford, J. (1995). Itinerarios transculturales. Barcelona: Gedisa.

Collins, R. (2004). Interaction Ritual Chains. Princeton y Oxford: Princeton University Press.

Crossley, N. (2001). The social body: Habit, identity and desire. London: Sage.

Csordas, T. (1990). Embodiment as a Paradigm for Anthropology. Ethos, 18 (1), 5-47.

Csordas, T. (1999). Embodiment and Cultural Phenomenology. In G. Weiss \& H. Fern Haber (Eds.). Perspectives on Embodiment (pp. 143-162). New York: Routledge.

Chase, L. (2008). Running big: Clydesdale runners and technologies of the body. Sociology of Sport Journal, 25, 130147.

D'Angelo, A. (2016). "Conciencia en la postura: el yoga entre el dualismo y la reflexividad". Runa. Archivo para las ciencias del hombre, 37 (1), 21-38.

Fine, G. (2012). Tiny Publics. A Theory of Group Action and Culture. New York: Sage.

Gelfman, N. (2017). Carreras shopping: un estudio de la práctica del running en Buenos Aires. Tesis de Maestría. Recuperada en RIDAA-UNQ Repositorio Institucional Digital de Acceso Abierto de la Universidad Nacional de Quilmes. https://ridaa.unq.edu.ar/handle/20.500.11807/269?show=full

Gil, G. J. (2002). Fútbol e identidades locales. Dilemas y conflictos latentes en una ciudad "feliz". Buenos Aires: Miño y Dávila.

Gil, G. J. (2006) Controles etnográficos y expertos en el campo: cuando los «nativos» nos leen. Cuadernos del Instituto Nacional de Antropología y Pensamiento Latinoamericano, 20, 129-148.

Gil, G. J. (2018). Deporte y estilos de vida. El running en Argentina. Antipoda. Revista de Antropología y Arqueología, 30, 43-63. https://dx.doi.org/10.7440/antipoda30.2018.03

Gil, Gastón Julián (2019). Correr y competir. Rituales de interacción y estilo de vida en el running". Revista del Museo de Antropología, 12 (1), 105-116. https://doi.org/10.31048/1852.4826.v12.n1.22020

Gil, G. J. (2020). Moralidad, virtud y emociones en el running. Aproximaciones etnográficas de un estilo de vida en la Argentina contemporánea. Sociologia \& Antropologia 10(2), 537-559. https://doi.org/10.1590/2238-38752 020v1029

Hannerz, U. (2003). Being there... and there... and there!". Ethnography, 4(2), 201-216.

Hanold, M. (2016). Ultrarunning. Space, place, and social experience. In W. Bridel, P. Markula \& J. Denison (Eds.). Endurance Running. A socio-cultural examination (pp. 181-195). New York: Routldege.

Hijós, M. (2017). "Fitters", "paleo" y "veggies": nuevas formas de clasificar lo comestible. Educación Física y Ciencia, 19(2), e034. https://doi.org/10.24215/23142561e 034.

Hijós, M. (2019). La carrera de los runners: una etnografia en Nike+ Run Club de Buenos Aires". Tesis de Maestría inédita. Posgrado en Antropología Social IDES-IDAES/UNSAM, Buenos Aires.

Howe, P. (2004). Sport, professionalism, and pain: Ethnographies of injury and risk. London: Routledge.

Howe, P. \& Morris, C. (2009). An Exploration of the Co-production of Performance Running Bodies and Natures Within Running Taskscapes. Journal of Sport \& Social Issues, 33 (3), 308-330.

Illouz, E. (2007). Intimidades congeladas. Las emociones en el capitalismo. Buenos Aires: Katz.

Ingold, T. (2000). The Perception of the Environment: Essays on Livelihood, Dwelling and Skill. London: Routledge.

Iragola Cairoli, V. (2019). Running: autogestión de una corporalidad emprendedora. Revista Latinoamericana de Estudios sobre Cuerpos, Emociones y Sociedad (RELACES), 10 (28), 39-51.

Lasalle, G.; Recours, R. \& Griffet, J. (2019). Cultures of running: characteristics and determinants. Sport in Society, 22(3), 342-360.

Latour, B. (2008). Reensamblar lo social. Una introducción a la teoría del actor-red. Buenos Aires: Manantial.

Le Breton, D. (1995). Antropología del cuerpo y modernidad. Buenos Aires: Nueva Visión.

Lewin, G. \& Puglisi, R. (2012). Acelerados en calma. Un análisis comparativo del tai chi y las técnicas meditativas de origen hindú en el contexto posmoderno. In S. Citro \& P. Ascheri (Eds.). Cuerpos en movimiento. Antropología de y desde las danzas (pp. 253-263). Buenos Aires: Biblos. 
Marcus, G. (1995). Ethnography in/of the World System: The Emergence of Multi-Sited Ethnography. Annual Review of Anthropology, 24, 95-117.

Mauss, M. (1991). Sociología y Antropología. Madrid: Tecnos.

Paterson, M. (2009). Haptic geographies: Ethnography, haptic knowledges and sensuous dispositions. Progress in Human Geography, 33(6), 766-788.

Reed-Danahay, D. (1997). Leaving Home: Schooling Stories and the Ethnography in Rural France. In D. ReedDanahay (Ed.). Auto/ethnography. Rewriting the Self and the Social (pp. 123-144). Oxford: Berg,

Robben, A. (1995). Seduction and Persuasion: The Politics of Truth and Emotion among Victims and Perpetrators of Violence. In A. Robben \& C. Nordstrom (Eds.). Fieldwork under Fire. Contemporary Studies of Violence Survival (pp. 81-103). Berkeley: University of California Press.

Shipway, R. \& Holloway, I. (2016). Health and the running body: Notes from an ethnography. International Review for the Sociology of Sport, 51(1), 78-96.

Shipway R.; Holloway, I. \& Jones, I. (2013). Organisations, practices, actors, and events: Exploring inside the distance running social world. International Review for the Sociology of Sport, 48(3), 259-276.

Wacquant, L. (2006). Entre las cuerdas. Cuadernos de un aprendiz de boxeador. Buenos Aires: Siglo XXI.

\section{Notas}

1 Ello llega incluso representarse ocasionalmente en el programa ESPN Run, emisión televisiva en la que, aunque los cuerpos presentados estén tonificados y sean apropiados para completar carreras de larga distancia, no se proyecta una imagen estética ideal del corredor "serio". Por el contrario, en más de un episodio el protagonista está lejos de mostrar la configuración corporal "perfecta", de delgadez, tonificación muscular y liviandad. Por ejemplo, en el programa dedicado al maratón de Bermuda, Alexandria plantea expresamente que concibe su "misión" en el running como un modo "para que todos sepan que los corredores somos todos de tamaños diferentes, de colores diferentes". Y ni siquiera se trata de un caso excepcional de corredores más robustos que protagonizan las emisiones semanales del ciclo. Ese programa pone en escena, cada vez con mayor énfasis y frecuencia, cuerpos "no hegemónicos" de corredores "serios" que asumen y cumplen desafíos relevantes, como correr varias maratones por año en tiempos destacados para un amateur.

2 Aunque no se trata de un término empleado de manera frecuente en el running en la Argentina, "carrera de larga distancia" hace referencia a las competencias que requieren un número considerable de kilómetros para ser completadas, por lo general a partir de 10 kilómetros.

3 El Cruce es responsabilidad del Club de Corredores, una entidad que organiza diversas carreras, tanto urbanas como de trail, además de ofrecer una amplia diversidad de servicios vinculados con el mundo del running (venta de indumentaria, asesoría médica y nutricional). Su formato fue variando desde su primera edición en 2002 cuando los 300 participantes debían montar sus propias carpas en los campamentos entre cada etapa. En la actualidad, varios miles de participantes de cada edición llegan luego de las primeras dos etapas a un campamento en el que todos los servicios necesarios (carpas, alimentación, baños químicos, servicios pagos de masajistas) están garantizados por la organización. Esta carrera constituye toda una marca en el país y en el exterior y reúne corredores de todos los rincones del mundo. Los despliegues organizativos suelen involucrar un par de helicópteros, drones con cámaras y una importante logística de traslado de micros de las líneas de largada y llegada hacia los campamentos.

4 El trailrunning constituye un género distintivo de carreras, en contraste con las más visibles, que son las urbanas (como los maratones). Gran parte de esas carreras "de aventura" se realizan en zonas de montaña, cordilleranas y precordilleranas, pero también en áreas rurales y hasta en pasajes costeros.

5 Precisamente, la costumbre de acompañar musicalmente el entrenamiento es un punto "conflictivo" en la metafísica del runner. Tal vez mucho más utilizado por corredores ocasionales o por quienes no están volcados completamente en el universo del running, suele ser desaconsejado por los expertos, muchos de los que consideran que la música se interpone en la necesidad de lograr una conexión mental y corporal con el medio ambiente. Aunque en ocasiones, no pocos runners los toman como un instrumento imprescindible en los entrenamientos largos, exigentes y repetitivos a los que se suelen someter. En última instancia, será la fenomenología de cada runner la que le dicte el mejor modo de experimentar sus placeres sensoriales, sea o no a partir de una armonía con la naturaleza (por ejemplo, extrayendo energía de las montañas, los lagos o la playa), o interfiriéndola con la música.

6 En la comedia cinematográfica Run Fatboy Run (2006), el protagonista (interpretado por Simon Pegg) literalmente atraviesa un muro de ladrillos en el Maratón de Londres. 